\title{
Looking for a new antitubercular pharmacophore site: synthesis and bioactivity of spiroheterocycles 2,3',4'-tri-subsituted-1,2- dihydro-4H,4' $H$-spiro[isoquinoline-3,5'-isoxazol]-4-ones
}

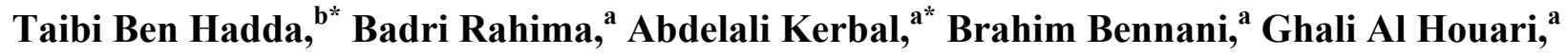 \\ Bouchra Filali Baba, ${ }^{a}$ Mehmet Akkurt, ${ }^{c}$ Gilles Demailly, ${ }^{d}$ and Mohammed Benazza ${ }^{d}$ \\ ${ }^{a}$ Laboratoire de Chimie Organique, Faculté des Sciences Dhar El Mehraz, Fès, Morocco \\ ${ }^{b}$ Laboratoire Chimie des Matériaux, Faculté des Sciences, Oujda, Morocco \\ ${ }^{c}$ Department of Physics, Faculty of Arts and Sciences, 38039 Kayseri, Turkey \\ ${ }^{d}$ Laboratoire des Glucides UMR 6219, Université de Picardie Jules Verne, 33 Rue Saint-Leu, \\ 80039 Amiens Cedex, France \\ E-mails: kerbalabdelali@yahoo.fr, tbenhadda@yahoo.fr
}

\begin{abstract}
Synthesis of a series of fourteen novel 2,3',4'-tri-subsituted-1,2-dihydro-4H,4' $H$ spiro[isoquinoline-3,5'-isoxazol]-4-ones was accomplished in good yield by regio and stereoselective 1,3-dipolar cycloaddition of $p$ - $\mathrm{R}^{2}$-benzadoxime 4-8 with dipolarophiles (3Z)-3[(4-substituted-phenyl)methylidene]-2-phenyl-2,3-dihydroisoquinolin-4(1H)-ones $\mathbf{1 - 3}$. The structure of the isolated products 9-23 was established through different spectroscopic techniques. X-Ray crystal structure analysis of one of the products confirms the structure and the selective region and stereochemistry of this cycloaddition. Their antitubercular activity is also evaluated.
\end{abstract}

Keywords: Spiroisoxazolines, 1,3-dipolar cycloaddition, antitubercular activity

\section{Introduction}

The spiroisoxazolines derivatives have emerged in recent years as candidates for drugs due to their herbicidal, plant-growth regulatory and antitumor activity. ${ }^{1,2}$ We have recently investigated the antitubercular activity of some spiroisoxazolines derivatives. ${ }^{3,4}$ With other kind of drugs we had also performed antimicrobial screening of 3-nitrozo-imidazo[1,2-a]pyrimidines. We had shown that compounds bearing a formyl, hydroxyl or nitroso side chains in 3-position are highly active as antitubercular (MIC $<6.25 \mu \mathrm{g} / \mathrm{mL} ; 98 \%$ Inhib.) ${ }^{5}$ and antibacterial agents (Gram+ and Gram-). ${ }^{6}$ 
General structure/activity relationship observations allowed us to suggest that functionalized side chain(s) like $[\mathrm{O}=\mathrm{C}-\mathrm{C}-\mathrm{O}],[\mathrm{O}=\mathrm{C}-\mathrm{C}-\mathrm{O}-\mathrm{N}]$ or $[\mathrm{X}-\mathrm{C}-\mathrm{O}]$ and $[\mathrm{X}-\mathrm{C}-\mathrm{O}-\mathrm{N}](\mathrm{X}=\mathrm{O}, \mathrm{S})$ are usually crucial for the diversity of the bioactivity (Figure 1).
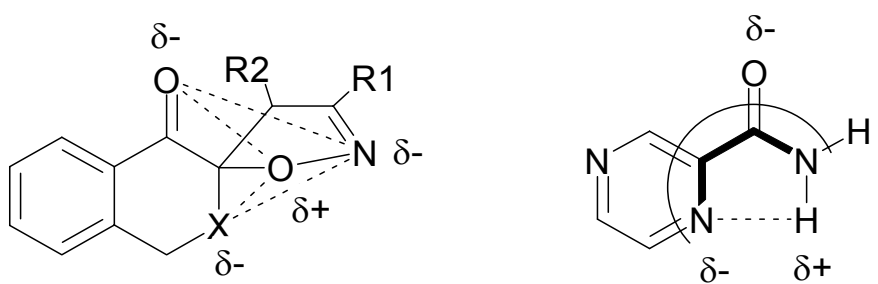

$$
(\mathrm{X}=\mathrm{O}, \mathrm{S})
$$

A : - Antitubercular agent ${ }^{3}$ - Anti-HIV agent ${ }^{4}$

B : Antitubercular agent
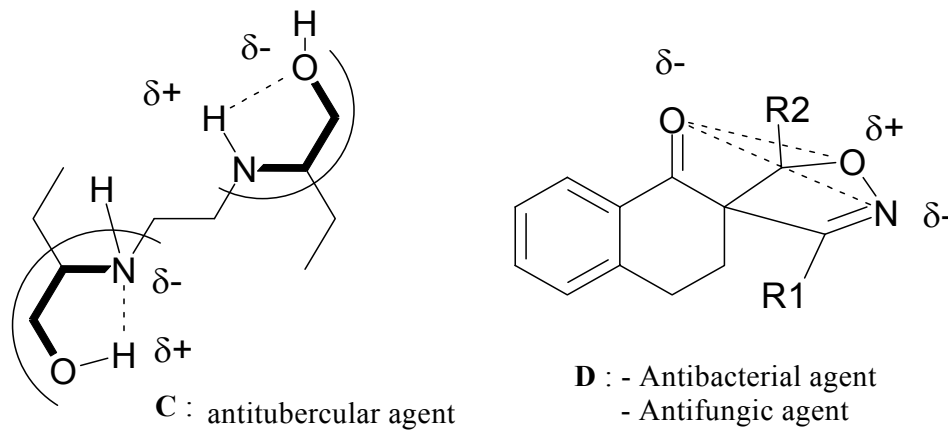

Figure 1. Structures of clinical antitubercular agents $(\mathbf{B}, \mathbf{C})$ and spiroheterocycles $(\mathbf{A}, \mathbf{D})$.

In our earlier studies of the 1,3-dipolar cycloaddition field, we had investigated the reaction of diarylnitrilimines with endocyclic dipolarophiles such as dihydroquinoline, ${ }^{7}$ indene ${ }^{8}$ or 3methoxycarbonyl-4H-1-benzopyran-4-one. ${ }^{9}$ We also studied the regio and stereochemistry of the reaction of diarylnitrilimines with the 2 -arylidene-indan-1-ones, ${ }^{10} 3$-arylidene-tetraline-4ones, ${ }^{11,12} 3$-arylidene-isothiochroman-4-ones ${ }^{13}$ and recently the 3-toluidene-2,3-dihydro-4(1H)isoquinolone with exocyclic dipolarophile groups. ${ }^{14}$

In our ongoing program, we report an efficient and short synthesis of new spiroheterocycles in good yields from 1,3-dipolar cycloaddition of dipolarophiles with the appropriate $p$ - $\mathrm{R}^{2}$ benzadoxime. The pharmacological investigations as antitubercular activity are also discussed.

\section{Results and Discussion}

\section{Chemistry}

The precursors or dipolarophiles (1-3); (3Z)-3-[(4-substituted-phenyl)methylidene]-2-phenyl-2,3dihydroisoquinolin-4(1H)-ones were prepared from para-substituted benzaldehydes, and 2- 
phenyl-2,3-dihydroisoquinolin-4(1H)-one as reactants. ${ }^{15}$ The cycloadditions of the dipolarophiles 1-3 with the $p-\mathrm{R}^{2}$-benzadoxime 4-8 were completely regioselective and stereoselective, to the extent that no other cycloadducts were detected in NMR spectra of the crude product mixtures. This may be attributed to steric effects controlling the reactions, such that the nitrogen atom of the NOH group of the compounds 4-8 becomes bonded to the most substituted olefinic carbon of the dipolarophile 1-3. Modest to good yields of the spiroheterocycles 9-23 were obtained (40$80 \%$ ) as shown in Scheme 1.<smiles>[R1]c1ccc(/C=C2/C(=O)c3ccccc3CN2c2ccccc2)cc1</smiles>

1-3
2: $\mathrm{R} 1=\mathrm{CH}_{3}$ 3: $\mathrm{R} 1=\mathrm{OCH}_{3}$<smiles>[R2]c1ccc(/C=N/O)cc1</smiles>

4-8

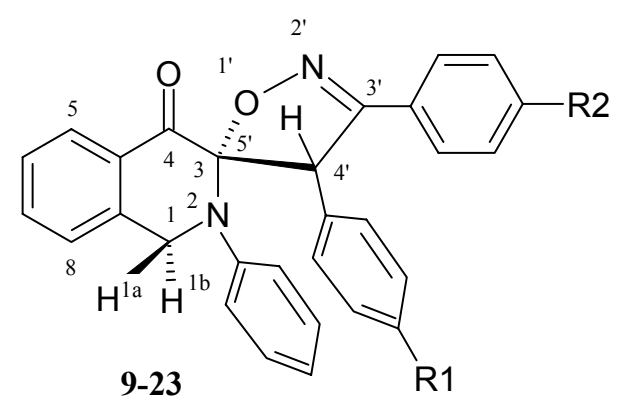

9: $(\mathrm{R} 1, \mathrm{R} 2)=(\mathrm{H}, \mathrm{H})$

10: $(\mathrm{R} 1, \mathrm{R} 2)=\left(\mathrm{H}_{,} \mathrm{CH}_{3}\right)$

11: $(\mathrm{R} 1, \mathrm{R} 2)=\left(\mathrm{H}, \mathrm{OCH}_{3}\right)$

12: $(\mathrm{R} 1, \mathrm{R} 2)=(\mathrm{H}, \mathrm{Cl})$

13: $(\mathrm{R} 1, \mathrm{R} 2)=\left(\mathrm{H}_{1} \mathrm{NO}_{2}\right)$

14: $(\mathrm{R} 1, \mathrm{R} 2)=\left(\mathrm{CH}_{3}, \mathrm{H}\right)$

15: $(\mathrm{R} 1, \mathrm{R} 2)=\left(\mathrm{CH}_{3}, \mathrm{CH}_{3}\right)$

16: $(\mathrm{R} 1, \mathrm{R} 2)=\left(\mathrm{CH}_{3}, \mathrm{OCH}_{3}\right)$
17: $(\mathrm{R} 1, \mathrm{R} 2)=\left(\mathrm{CH}_{3}, \mathrm{Cl}\right)$

18: $(\mathrm{R} 1, \mathrm{R} 2)=\left(\mathrm{CH}_{3}, \mathrm{NO}_{2}\right)$

19: $(\mathrm{R} 1, \mathrm{R} 2)=\left(\mathrm{OCH}_{3}, \mathrm{H}\right)$

20: $(\mathrm{R} 1, \mathrm{R} 2)=\left(\mathrm{OCH}_{3}, \mathrm{CH}_{3}\right)$

21: $(\mathrm{R} 1, \mathrm{R} 2)=\left(\mathrm{OCH}_{3}, \mathrm{OCH}_{3}\right)$

22: $(\mathrm{R} 1, \mathrm{R} 2)=\left(\mathrm{OCH}_{3}, \mathrm{Cl}\right)$

23: $(\mathrm{R} 1, \mathrm{R} 2)=\left(\mathrm{OCH}_{3}, \mathrm{NO}_{2}\right)$

Scheme 1. Synthesis of spiroheterocycles 9-23.

The cycloaddition could also taken place in toluene and triethylamine. All compounds were characterized using ${ }^{1} \mathrm{H}$ and ${ }^{13} \mathrm{C}$ NMR data's which are in agreement with previously reported similar compounds. ${ }^{13,16,17}$ The selective NMR data are represented in Tables 1 and 2.

The crystallographic structure of compound $\mathbf{1 8}$ has been previously deteremined. ${ }^{18}$ The ${ }^{1} \mathrm{H}$ NMR and crystallographic data of compound 18, for example, show that the 1,3-dipolar cycloaddition occurs with inverted regiochemistry to that obtained with chromanone. ${ }^{18}$ The same observation have been noted elsewhere for similar other spiroheterocycles. ${ }^{19}$ 
Table 1. Selected IR and ${ }^{1} \mathrm{H}$ NMR data of compounds 9-23

\begin{tabular}{|c|c|c|c|c|c|c|c|}
\hline Comp. & $(\mathrm{R} 1, \mathrm{R} 2)$ & $\begin{array}{c}\mathrm{IR} \\
(\mathrm{v} \mathrm{C}=\mathrm{O})\end{array}$ & $\begin{array}{l}\delta \mathrm{R} 1 \\
\delta \mathrm{R} 2 \\
\end{array}$ & $\begin{array}{l}\delta \mathrm{H}^{1 \mathrm{a}} \\
\delta \mathrm{H}^{1 \mathrm{~b}} \\
\end{array}$ & $\delta \mathrm{H}^{4^{\prime}}$ & $\begin{array}{c}\delta \mathrm{H} \\
\text { (Aroma) }\end{array}$ & $\delta \mathrm{H}^{5}$ \\
\hline \multirow{3}{*}{9} & \multirow{3}{*}{$(\mathrm{H}, \mathrm{H})$} & \multirow{3}{*}{1703} & - & $4.05(\mathrm{~d}, 1 \mathrm{H})$ & \multirow{3}{*}{$6.15(\mathrm{~s}, 1 \mathrm{H})$} & $6.70-7.75$ & $8.25(\mathrm{dd}, 1 \mathrm{H})$ \\
\hline & & & - & $5.05(\mathrm{~d}, 1 \mathrm{H})$ & & \multirow[t]{2}{*}{$(\mathrm{m}, 18 \mathrm{H})$} & ${ }^{4} \mathrm{~J}=1.25$ \\
\hline & & & & ${ }^{2} \mathrm{~J}=17.35$ & & & ${ }^{3} \mathrm{~J}=7.13$ \\
\hline \multirow{3}{*}{10} & \multirow{3}{*}{$\left(\mathrm{H}, \mathrm{CH}_{3}\right)$} & \multirow{3}{*}{1686} & - & $4.00(\mathrm{~d}, 1 \mathrm{H})$ & \multirow{3}{*}{$6.05(\mathrm{~s}, 1 \mathrm{H})$} & $6.55-7.55$ & $8.15(\mathrm{dd}, 1 \mathrm{H})$ \\
\hline & & & $2.25(\mathrm{~s}, 3 \mathrm{H})$ & $5.00(\mathrm{~d}, 1 \mathrm{H})$ & & \multirow{2}{*}{$(\mathrm{m}, 17 \mathrm{H})$} & ${ }^{4} \mathrm{~J}=1.25$ \\
\hline & & & & ${ }^{2} \mathrm{~J}=17.20$ & & & ${ }^{3} \mathrm{~J}=7.70$ \\
\hline \multirow{3}{*}{11} & \multirow{3}{*}{$\left(\mathrm{H}, \mathrm{OCH}_{3}\right)$} & \multirow{3}{*}{1693} & - & $4.00(\mathrm{~d}, 1 \mathrm{H})$ & \multirow{3}{*}{$6.00(\mathrm{~s}, 1 \mathrm{H})$} & \multirow{3}{*}{$\begin{array}{l}6.70-7.50 \\
(\mathrm{~m}, 17 \mathrm{H})\end{array}$} & $8.20(\mathrm{dd}, 1 \mathrm{H})$ \\
\hline & & & $3.70(\mathrm{~s}, 3 \mathrm{H})$ & $4.95(\mathrm{~d}, 1 \mathrm{H})$ & & & ${ }^{4} \mathrm{~J}=1.30$ \\
\hline & & & & ${ }^{2} \mathrm{~J}=17.60$ & & & ${ }^{3} \mathrm{~J}=7.60$ \\
\hline \multirow{3}{*}{12} & \multirow{3}{*}{$(\mathrm{H}, \mathrm{Cl})$} & \multirow{3}{*}{1685} & - & $4.00(\mathrm{~d}, 1 \mathrm{H})$ & \multirow{3}{*}{$6.05(\mathrm{~s}, 1 \mathrm{H})$} & \multirow{3}{*}{$\begin{array}{c}6.50-7.55 \\
(\mathrm{~m}, 17 \mathrm{H})\end{array}$} & $8.20(\mathrm{dd}, 1 \mathrm{H})$ \\
\hline & & & - & $4.95(\mathrm{~d}, 1 \mathrm{H})$ & & & ${ }^{4} \mathrm{~J}=1.35$ \\
\hline & & & & ${ }^{2} \mathrm{~J}=17.20$ & & & ${ }^{3} \mathrm{~J}=7.80$ \\
\hline \multirow{3}{*}{13} & \multirow{3}{*}{$\left(\mathrm{H}, \mathrm{NO}_{2}\right)$} & \multirow{3}{*}{1706} & - & $3.85(\mathrm{~d}, 1 \mathrm{H})$ & \multirow{3}{*}{$6.00(\mathrm{~s}, 1 \mathrm{H})$} & \multirow{3}{*}{$\begin{array}{c}6.50-7.60 \\
(\mathrm{~m}, 17 \mathrm{H})\end{array}$} & $8.15(\mathrm{dd}, 1 \mathrm{H})$ \\
\hline & & & - & $4.60(\mathrm{~d}, 1 \mathrm{H})$ & & & ${ }^{4} \mathrm{~J}=1.65$ \\
\hline & & & & ${ }^{2} \mathbf{J}=17.25$ & & & ${ }^{3} \mathrm{~J}=7.80$ \\
\hline \multirow{3}{*}{14} & & & $2.20(\mathrm{~s}, 3 \mathrm{H})$ & $4.75(\mathrm{~d}, 1 \mathrm{H})$ & & $6.90-7.50$ & $7.60(\mathrm{dd}, 1 \mathrm{H})$ \\
\hline & $\left(\mathrm{CH}_{3}, \mathrm{H}\right)$ & 1694 & - & $4.80(\mathrm{~d}, 1 \mathrm{H})$ & $6.00(\mathrm{~s}, 1 \mathrm{H})$ & $(\mathrm{m}, 17 \mathrm{H})$ & ${ }^{4} \mathrm{~J}=1.20$ \\
\hline & & & & ${ }^{2} \mathbf{J}=17.00$ & & & ${ }^{3} \mathrm{~J}=7.35$ \\
\hline & & & $2.20(\mathrm{~s}, 3 \mathrm{H})$ & $4.10(\mathrm{~d}, 1 \mathrm{H})$ & & $6.45-7.55$ & $8.20(\mathrm{dd}, 1 \mathrm{H})$ \\
\hline 15 & $\left(\mathrm{CH}_{3}, \mathrm{CH}_{3}\right)$ & 1703 & $2.30(\mathrm{~s}, 3 \mathrm{H})$ & $5.05(\mathrm{~d}, 1 \mathrm{H})$ & $6.05(\mathrm{~s}, 1 \mathrm{H})$ & $(\mathrm{m}, 16 \mathrm{H})$ & ${ }^{4} \mathrm{~J}=1.25$ \\
\hline & & & & ${ }^{2} \mathbf{J}=17.25$ & & & ${ }^{3} \mathrm{~J}=7.80$ \\
\hline & & & $2.15(\mathrm{~s}, 3 \mathrm{H})$ & $4.10(\mathrm{~d}, 1 \mathrm{H})$ & & $6.50-7.55$ & $8.20(\mathrm{dd}, 1 \mathrm{H})$ \\
\hline 16 & $\left(\mathrm{CH}_{3}, \mathrm{OCH}_{3}\right)$ & 1704 & $3.75(\mathrm{~s}, 3 \mathrm{H})$ & $5.05(\mathrm{~d}, 1 \mathrm{H})$ & $6.00(\mathrm{~s}, 1 \mathrm{H})$ & $(\mathrm{m}, 16 \mathrm{H})$ & ${ }^{4} \mathrm{~J}=1.15$ \\
\hline & & & & ${ }^{2} \mathbf{J}=17.30$ & & & ${ }^{3} \mathrm{~J}=7.70$ \\
\hline & & & $2.20(\mathrm{~s}, 3 \mathrm{H})$ & $4.10(\mathrm{~d}, 1 \mathrm{H})$ & & $6.40-7.55$ & $8.20(\mathrm{dd}, 1 \mathrm{H})$ \\
\hline 17 & $\left(\mathrm{CH}_{3}, \mathrm{Cl}\right)$ & 1710 & - & $5.05(\mathrm{~d}, 1 \mathrm{H})$ & $6.05(\mathrm{~s}, 1 \mathrm{H})$ & $(\mathrm{m}, 16 \mathrm{H})$ & ${ }^{4} \mathrm{~J}=1.45$ \\
\hline & & & & ${ }^{2} \mathbf{J}=17.25$ & & & ${ }^{3} \mathrm{~J}=7.75$ \\
\hline & & & $2.15(\mathrm{~s}, 3 \mathrm{H})$ & $4.00(\mathrm{~d}, 1 \mathrm{H})$ & & $6.35-7.75$ & $8.15(\mathrm{dd}, 1 \mathrm{H})$ \\
\hline 18 & $\left(\mathrm{CH}_{3}, \mathrm{NO}_{2}\right)$ & 1708 & - & $4.95(\mathrm{~d}, 1 \mathrm{H})$ & $6.10(\mathrm{~s}, 1 \mathrm{H})$ & $(\mathrm{m}, 16 \mathrm{H})$ & ${ }^{4} \mathrm{~J}=1.45$ \\
\hline & & & & ${ }^{2} \mathbf{J}=17.35$ & & & ${ }^{3} \mathrm{~J}=7.80$ \\
\hline & & & $3.85(\mathrm{~s}, 3 \mathrm{H})$ & $4.10(\mathrm{~d}, 1 \mathrm{H})$ & & $6.50-7.65$ & $8.05(\mathrm{dd}, 1 \mathrm{H})$ \\
\hline 19 & $\left(\mathrm{OCH}_{3}, \mathrm{H}\right)$ & 1697 & - & $5.05(\mathrm{~d}, 1 \mathrm{H})$ & $6.00(\mathrm{~s}, 1 \mathrm{H})$ & $(\mathrm{m}, 17 \mathrm{H})$ & ${ }^{4} \mathrm{~J}=1.40$ \\
\hline & & & & ${ }^{2} \mathbf{J}=17.30$ & & & ${ }^{3} \mathrm{~J}=7.25$ \\
\hline & & & $3.65(\mathrm{~s}, 3 \mathrm{H})$ & $4.10(\mathrm{~d}, 1 \mathrm{H})$ & & $6.35-7.55$ & $8.20(\mathrm{dd}, 1 \mathrm{H})$ \\
\hline 20 & $\left(\mathrm{OCH}_{3}, \mathrm{CH}_{3}\right)$ & 1691 & $2.30(\mathrm{~s}, 3 \mathrm{H})$ & $5.05(\mathrm{~d}, 1 \mathrm{H})$ & $6.05(\mathrm{~s}, 1 \mathrm{H})$ & $(\mathrm{m}, 16 \mathrm{H})$ & ${ }^{4} \mathrm{~J}=1.40$ \\
\hline & & & & ${ }^{2} \mathbf{J}=17.30$ & & & ${ }^{3} \mathrm{~J}=7.70$ \\
\hline & & & $3.65(\mathrm{~s}, 3 \mathrm{H})$ & $4.10(\mathrm{~d}, 1 \mathrm{H})$ & & $6.35-7.55$ & $8.15(\mathrm{dd}, 1 \mathrm{H})$ \\
\hline
\end{tabular}




\begin{tabular}{|c|c|c|c|c|c|c|c|}
\hline 21 & $\left(\mathrm{OCH}_{3}, \mathrm{OCH}_{3}\right)$ & 1698 & $3.75(\mathrm{~s}, 3 \mathrm{H})$ & $\begin{array}{c}5.05(\mathrm{~d}, 1 \mathrm{H}) \\
{ }^{2} \mathrm{~J}=17.30\end{array}$ & $6.00(\mathrm{~s}, 1 \mathrm{H})$ & $(\mathrm{m}, 16 \mathrm{H})$ & $\begin{aligned}{ }^{4} \mathrm{~J} & =1.35 \\
{ }^{3} \mathrm{~J} & =7.60\end{aligned}$ \\
\hline 22 & $\left(\mathrm{OCH}_{3}, \mathrm{Cl}\right)$ & 1695 & $\begin{array}{c}3.65(\mathrm{~s}, 3 \mathrm{H}) \\
-\end{array}$ & $\begin{array}{c}4.10(\mathrm{~d}, 1 \mathrm{H}) \\
5.05(\mathrm{~d}, 1 \mathrm{H}) \\
{ }^{2} \mathrm{~J}=17.30\end{array}$ & $6.05(\mathrm{~s}, 1 \mathrm{H})$ & $\begin{array}{l}6.35-7.60 \\
(\mathrm{~m}, 16 \mathrm{H})\end{array}$ & $\begin{array}{c}8.20(\mathrm{dd}, 1 \mathrm{H}) \\
{ }^{4} \mathrm{~J}=1.45 \\
{ }^{3} \mathrm{~J}=7.80\end{array}$ \\
\hline 23 & $\left(\mathrm{OCH}_{3}, \mathrm{NO}_{2}\right)$ & 1705 & $\begin{array}{c}3.65(\mathrm{~s}, 3 \mathrm{H}) \\
-\end{array}$ & $\begin{array}{c}4.05(\mathrm{~d}, 1 \mathrm{H}) \\
4.95(\mathrm{~d}, 1 \mathrm{H}) \\
{ }^{2} \mathrm{~J}=17.35\end{array}$ & $6.10(\mathrm{~s}, 1 \mathrm{H})$ & $\begin{array}{l}6.40-7.75 \\
(\mathrm{~m}, 16 \mathrm{H})\end{array}$ & $\begin{array}{c}8.15(\mathrm{dd}, 1 \mathrm{H}) \\
{ }^{4} \mathrm{~J}=1.30 \\
{ }^{3} \mathrm{~J}=7.65\end{array}$ \\
\hline
\end{tabular}

Table 2. Selected ${ }^{13} \mathrm{C}$ NMR data of compounds $\mathbf{9 - 2 3}$

\begin{tabular}{cccccccc}
\hline Comp. & $(\mathrm{R} 1, \mathrm{R} 2)$ & $\begin{array}{c}\delta \mathrm{C} \\
(\mathrm{R} 1)\end{array}$ & $\delta \mathrm{C}(\mathrm{R} 2)$ & $\begin{array}{c}\delta \mathrm{C}^{1} \\
\left(\mathrm{NCH}_{2}-\right)\end{array}$ & $\begin{array}{c}\delta \mathrm{C}^{3,5^{\prime}} \\
(\text { spiro-C })\end{array}$ & $\begin{array}{c}\delta \mathrm{C}^{4} \\
(-\mathrm{C}=\mathrm{O})\end{array}$ & $\begin{array}{c}\delta \mathrm{C}^{4^{\prime}} \\
(-\underline{\mathrm{CH}}-)\end{array}$ \\
\hline $\mathbf{9}$ & $(\mathrm{H}, \mathrm{H})$ & - & - & 55.40 & 102.40 & 187.25 & 53.70 \\
$\mathbf{1 0}$ & $(\mathrm{H}, \mathrm{CH})$ & - & 21.50 & 55.34 & 102.40 & 187.65 & 54.50 \\
$\mathbf{1 1}$ & $\left(\mathrm{H}, \mathrm{OCH}_{3}\right)$ & - & 54.70 & 55.35 & 102.20 & 187.85 & 55.30 \\
$\mathbf{1 2}$ & $(\mathrm{H}, \mathrm{Cl})$ & - & - & 55.35 & 103.00 & 187.35 & 54.30 \\
$\mathbf{1 3}$ & $\left(\mathrm{H}_{2} \mathrm{NO}_{2}\right)$ & - & - & 55.30 & 102.80 & 187.80 & 54.00 \\
$\mathbf{1 4}$ & $\left(\mathrm{CH}_{3}, \mathrm{H}\right)$ & 21.15 & - & 52.15 & 103.95 & 187.35 & 57.40 \\
$\mathbf{1 5}$ & $\left(\mathrm{CH}_{3}, \mathrm{CH}_{3}\right)$ & 21.10 & 21.50 & 55.35 & 102.40 & 187.80 & 54.35 \\
$\mathbf{1 6}$ & $\left(\mathrm{CH}_{3}, \mathrm{OCH}_{3}\right)$ & 21.10 & 54.50 & 55.35 & 102.15 & 187.95 & 55.30 \\
$\mathbf{1 7}$ & $\left(\mathrm{CH}_{3}, \mathrm{Cl}\right)$ & 21.10 & - & 55.35 & 102.95 & 187.50 & 54.10 \\
$\mathbf{1 8}$ & $\left(\mathrm{CH}_{3}, \mathrm{NO}_{2}\right)$ & 21.10 & - & 55.40 & 103.95 & 187.00 & 53.70 \\
$\mathbf{1 9}$ & $\left(\mathrm{OCH}_{3}, \mathrm{H}\right)$ & 53.80 & - & 55.35 & 102.65 & 187.55 & 55.20 \\
$\mathbf{2 0}$ & $\left(\mathrm{OCH}_{3}, \mathrm{CH}\right)$ & 54.00 & 21.45 & 55.35 & 102.15 & 187.85 & 55.17 \\
$\mathbf{2 1}$ & $\left(\mathrm{OCH}_{3}, \mathrm{OCH}_{3}\right)$ & 54.15 & 55.20 & 55.35 & 101.90 & 187.00 & 55.30 \\
$\mathbf{2 2}$ & $\left(\mathrm{OCH}_{3}, \mathrm{Cl}\right)$ & 53.90 & - & 55.35 & 102.35 & 187.75 & 55.20 \\
$\mathbf{2 3}$ & $\left(\mathrm{OCH}_{3}, \mathrm{NO}_{2}\right)$ & 53.40 & - & 55.40 & 103.65 & 187.10 & 55.20 \\
\hline
\end{tabular}

\section{Antimycobacterial activity}

The antimycobacterial activity of the compounds was determined with the objective to identify the compounds having inhibitory activity against $M$. tuberculosis.

Interesting results were obtained from these assays and data is reported in Table 3 . The in vitro antimycobacterial activities of these compounds 9-23 were inferior to that of Isoniazid against $M$. tuberculosis $\mathrm{H}_{37} \mathrm{Rv}$. Further, the compounds 9-23 had either little or no activity (17-64\% inhibition). 
Table 3. Range of $\%$ inhibition values (MIC $>6.25 \mu \mathrm{g} / \mathrm{mL})$ of compounds 9-23 against Mycobacterium tuberculosis $\mathrm{H}_{37} \mathrm{Rv}$ strains

\begin{tabular}{|c|c|c|c|c|c|}
\hline $\begin{array}{l}\text { Compd ID } \\
\text { (TAACF ID) }\end{array}$ & (R1, R2) & $\begin{array}{c}\text { M.W. } \\
\text { (g/mole) }\end{array}$ & $\begin{array}{c}\mathrm{MIC} \\
(\mu \mathrm{g} / \mathrm{mL})\end{array}$ & $\begin{array}{c}\% \\
\text { Inhibition } \\
\text { (Alamar test) }\end{array}$ & Activity \\
\hline $\begin{array}{c}9 \\
(299937)\end{array}$ & $(\mathrm{H}, \mathrm{H})$ & 430 & $>6.25$ & 42 & negative \\
\hline $\begin{array}{c}10 \\
(299938)\end{array}$ & $\left(\mathrm{H}, \mathrm{CH}_{3}\right)$ & 444.5 & $>6.25$ & 19 & negative \\
\hline $\begin{array}{c}11 \\
(299939)\end{array}$ & $\left(\mathrm{H}, \mathrm{OCH}_{3}\right)$ & 460.5 & $>6.25$ & 44 & negative \\
\hline $\begin{array}{c}12 \\
(299940)\end{array}$ & $(\mathrm{H}, \mathrm{Cl})$ & 468.9 & $>6.25$ & 48 & negative \\
\hline $\begin{array}{c}13 \\
(299941)\end{array}$ & $\left(\mathrm{H}, \mathrm{NO}_{2}\right)$ & 477.5 & $>6.25$ & 24 & negative \\
\hline $\begin{array}{c}14 \\
(299942)\end{array}$ & $\left(\mathrm{CH}_{3}, \mathrm{H}\right)$ & 444.5 & $>6.25$ & 20 & negative \\
\hline $\begin{array}{c}15 \\
(299943)\end{array}$ & $\left(\mathrm{CH}_{3}, \mathrm{CH}_{3}\right)$ & 458.5 & $>6.25$ & 19 & negative \\
\hline $\begin{array}{c}16 \\
(299944)\end{array}$ & $\left(\mathrm{CH}_{3}, \mathrm{OCH}_{3}\right)$ & 474.5 & $>6.25$ & 42 & negative \\
\hline $\begin{array}{c}17 \\
(299945)\end{array}$ & $\left(\mathrm{CH}_{3}, \mathrm{Cl}\right)$ & 478.9 & $>6.25$ & 33 & negative \\
\hline $\begin{array}{c}18 \\
(299946)\end{array}$ & $\left(\mathrm{CH}_{3}, \mathrm{NO}_{2}\right)$ & 489.5 & $>6.25$ & 64 & negative \\
\hline $\begin{array}{c}19 \\
(299951)\end{array}$ & $\left(\mathrm{OCH}_{3}, \mathrm{H}\right)$ & 460.5 & $>6.25$ & 60 & negative \\
\hline $\begin{array}{c}\mathbf{2 0} \\
(299948)\end{array}$ & $\left(\mathrm{OCH}_{3}, \mathrm{CH}_{3}\right)$ & 474.5 & $>6.25$ & 49 & negative \\
\hline $\begin{array}{c}21 \\
(299949)\end{array}$ & $\left(\mathrm{OCH}_{3}, \mathrm{OCH}_{3}\right)$ & 490.5 & $>6.25$ & 34 & negative \\
\hline $\begin{array}{c}22 \\
(299950)\end{array}$ & $\left(\mathrm{OCH}_{3}, \mathrm{Cl}\right)$ & 494.9 & $>6.25$ & 53 & negative \\
\hline $\begin{array}{c}23 \\
(299947)\end{array}$ & $\left(\mathrm{OCH}_{3}, \mathrm{NO}_{2}\right)$ & 505.5 & $>6.25$ & 44 & negative \\
\hline
\end{tabular}

The replacement of the nitro substituent (R2) by a methyl or a methoxy group in phenyl ring of $\mathrm{C}\left(3^{\prime}\right)$ position, as in $\mathbf{1 0}$ and 11, respectively, did not cause any apparent change of activity (\% inhibition <90\%). Curiously, 4'-p-methylphenyl-, and 3'-p-nitrophenyl-substitution as in 18 $\left[(\mathrm{R} 1, \mathrm{R} 2)=\left(\mathrm{CH}_{3}, \mathrm{NO}_{2}\right)\right]$, abolished any activity indicating that not only specific electronic but 
also steric requirements are needed for activity $(\%$ Inhibition $=64 \%)$. This suggests that a bulky group or disubstitution on the $\mathrm{N}(2)$ and $\mathrm{C}(4$ ') positions are not favourable for antimycobacterial activity.

\section{Structure activity relationship}

The antimycobacterial activity data in Table 3 clearly show that the compounds 9-23 having an aryl substituent in $\mathrm{N}(2)$ position of the isoquinoline ring exhibited low activity against $M$. tuberculosis (Scheme 1).

All compounds 9-23 had an almost similar range of MIC values; they differ only by \% inhibition, in spite of having two different aryls at C(3) and C(4), as shown in Figure 2.

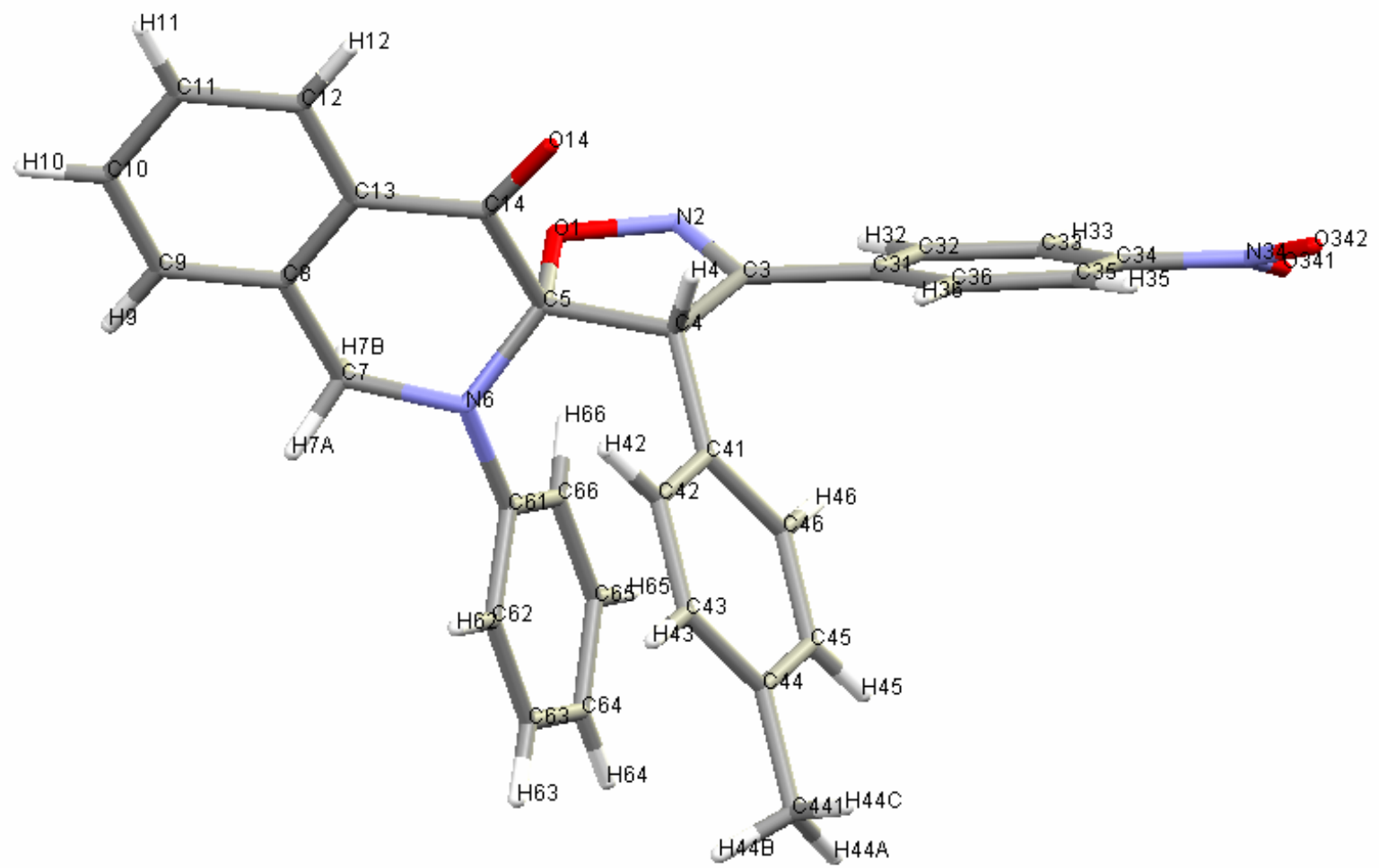

Figure 2. A view of compound (18) with the atom-numbering scheme. ${ }^{21}$ Distance: $\mathrm{O}(1)$--$\mathrm{O}(14)=3.03 \AA ; \mathrm{O}(1)---\mathrm{N}(6)=2.43 \AA ; \mathrm{O}(14)---\mathrm{N}(2)=3.62 \AA$. Angle: $\left(\mathrm{O}^{1}, \mathrm{C}^{5}, \mathrm{~N}^{6}\right)=112^{\circ}$; torsion angle: $\left(\mathrm{O}^{14}-\mathrm{C}^{14}-\mathrm{C}^{5}-\mathrm{O}^{1}\right)=89^{\circ} ;\left(\mathrm{O}^{14}-\mathrm{C}^{14}-\mathrm{C}^{3}-\mathrm{N}^{2}\right)=115^{\circ} ;\left(\mathrm{C}^{4}, \mathrm{C}^{3}, \mathrm{C}^{31}, \mathrm{C}^{36}\right)=20^{\circ}$; $\left(\mathrm{N}^{2}, \mathrm{C}^{3}, \mathrm{C}^{31}, \mathrm{C}^{32}\right)=23^{\circ} ;\left(\mathrm{O}^{341}, \mathrm{~N}^{34}, \mathrm{C}^{34}, \mathrm{C}^{33}\right)=14^{\circ}$.

It contains an almost planar isoxazoline ring. The $\mathrm{C}$ atoms of the isoquinoline moiety lie in a common plane, with the $\mathrm{N}$ atom deviating by 0.474 (2) $\AA$ from that plane. 


\section{Conclusions}

In this paper we report efficient and short synthesis of the spiroheterocycles 9-23 in good yields from 1,3-dipolar cycloaddition of dipolarophiles $N$-phenyl-3-( $p$ - $\mathrm{R}^{1}$-phenyl)-4-isoquinolone (1-3) with the appropriate $p$ - $\mathrm{R}^{2}$-benzadoxime (4-8). The more interesting result which could be emphasised is the inverted regio and stereochemistry of spirane ring formation in contrast with the result obtained in the literature with chromanone. This is likely due to the presence of the neighboring $\mathrm{N}$-aryl group. Due to the presence of rigid $\mathrm{O}=\mathrm{C}^{5}-\mathrm{O}^{1}-\mathrm{N}^{2}$ pharmacophore, anti-HIV screening studies are in progress at National Cancer Institute (NCI/ USA) in order to elucidate the structure/activity relationships.

\section{Experimental Section}

General Procedures. Melting points are measured on banc KOFLER without corrections. NMR spectra $\left({ }^{1} \mathrm{H},{ }^{13} \mathrm{C}\right)$ were recorded on a Bruker Avance (operating at $300 \mathrm{MHz}$ ). NMR data are listed in ppm and are reported relative to tetramethylsilane; residual solvent peaks being used as internal standard with external calibration. Infra-red spectra were recorded in $\mathrm{KBr}$ pellets using a Perkin-Elmer 1600 FT-IR spectrometer. Mass spectra were recorded on a Hewlett-Packard 5989A Mass Spectrometer $(70 \mathrm{eV})$ Nermag R 1010-C electronic impact and elemental analysis (CNRS, Université Paul Sabatier and Toulouse, France).

\section{Antitubercular activity}

Primary screening was conducted at $6.25 \mu \mathrm{g} \mathrm{mL}{ }^{-1}$ against $\mathrm{M}$. tuberculosis $\mathrm{H}_{37} \mathrm{Rv}$ (ATCC 27294) in BACTEC 12B medium using a broth microdilution assay, the Microplate Alamar Blue Assay (MABA) ${ }^{20}$ Compounds exhibiting fluorescence were tested in the BACTEC 460 radiometric system. Compounds demonstrating at least $90 \%$ inhibition in the primary screen were retested at lower concentrations against $\mathrm{M}$. tuberculosis $\mathrm{H}_{37} \mathrm{Rv}$ to determine the MIC using MABA. The MIC is defined as the lowest concentration effecting a reduction in fluorescence of $99 \%$ relative to controls. ${ }^{20}$

\section{General procedure of the preparation of spiroheterocycles 9-23}

A mixture of a dipolarophile $(\mathbf{1 - 3} ; \mathbf{6 . 8} \mathrm{mmol})$ and $p$ - $\mathrm{R}^{2}$-benzadoxime $(4-8 ; 7 \mathrm{mmol})$ was stirred in chloroform $(20 \mathrm{ml})$ at cold temperature $\left(-10^{\circ} \mathrm{C}\right)$. To this mixture was added $15 \mathrm{ml}$ of sodium hypochlorite (degree $=12 \mathrm{~g} \mathrm{NaClO}_{4} / 100 \mathrm{~g}$ solution) drop ways over $20 \mathrm{~min}$. The temperature was controlled and has not to excess $\left(-5^{\circ} \mathrm{C}\right)$. After complete addition of $\mathrm{NaOCl}$, the mixture was stirred 4-8 $\mathrm{h}$ at room temperature, until TLC indicated complete disappearance of reactants. The compounds were extracted by chloroform, washed by water and dried on sodium sulphate. The compounds 9-23 were obtained after evaporation and crystallisation in ethanol. 
2,3',4'-Tri-phenyl-1,2-dihydro-4H,4' $\boldsymbol{H}$-spiro[isoquinoline-3,5'-isoxazol]-4-one $\quad$ (9). This compound was obtained as white-yellow powder. Yield $70 \%$. M.p. $174-176{ }^{\circ} \mathrm{C}$. IR, $v$ $(\mathrm{C}=\mathrm{O}): 1703 \mathrm{~cm}^{-1} .{ }^{1} \mathrm{H}$ NMR: $4.05\left(\mathrm{~d}, 1 \mathrm{H}, \mathrm{H}^{1 \mathrm{a}},{ }^{2} \mathrm{JH}^{1 \mathrm{a}}-\mathrm{H}^{1 \mathrm{~b}}=17.35\right) ; 5.05\left(\mathrm{~d}, 1 \mathrm{H}, \mathrm{H}^{1 \mathrm{~b}},{ }^{2} \mathrm{JH}^{1 \mathrm{~b}}-\mathrm{H}^{1 \mathrm{a}}=\right.$ 17.35); $6.15\left(\mathrm{~s}, 1 \mathrm{H}, \mathrm{H}^{4}\right)$; 6.70-7.75 (m, 18H, Aromatic $\left.\mathrm{H}\right) ; 8.25\left(\mathrm{dd}, 1 \mathrm{H}, \mathrm{H}^{5},{ }^{4} \mathrm{~J}=1.25,{ }^{3} \mathrm{~J}=7.13\right)$. ${ }^{13} \mathrm{C}$ NMR: $55.40\left(\mathrm{~N}^{-} \mathrm{CH}_{2}{ }^{-}\right) ; 53.70\left(-\mathrm{C}^{4} \mathrm{H}-\right) ; 120.40$ (spiro-C); 126.10; 128.65; 132.30; 140.05; $140.45 ; 148.65 ; 159.60$ (quat. $\mathrm{C} \mathrm{sp}{ }^{2}$ ); $125.40 ; 125.65 ; 126.00 ; 126.35 ; 126.85 ; 127.00 ; 128.15$; 128.85; 128;95; 130.15; 134.00; (tert. C $\mathrm{sp}^{2}$ ). MS (EI, $\left.70 \mathrm{eV}\right):[\mathrm{M}]^{+} \mathrm{m} / \mathrm{z}: \mathrm{M}$ (430.5) $\left[\mathrm{C}_{29} \mathrm{H}_{22} \mathrm{~N}_{2} \mathrm{O}_{2}\right](15 \%) ; 118$ (100\%). Anal. Calcd. For $\mathrm{C}_{29} \mathrm{H}_{22} \mathrm{~N}_{2} \mathrm{O}_{2}$ : C, 80.91; H, 5.15; N, 6.51 . Found: C, 81.02; H, 4.98; N, 6.43.

\section{2-Phenyl-4'-phenyl-3' -(4-methylphenyl)-1,2-dihydro-4H,4' $H$-spiro[isoquinoline-3,5'-}

isoxazol]-4-one (10). This compound was obtained as white-yellow powder. Yield 72\%. M.p. 247-251 ${ }^{\circ} \mathrm{C}$. IR, $v(\mathrm{C}=\mathrm{O}): 1686 \mathrm{~cm}^{-1} .{ }^{1} \mathrm{H}$ NMR: $2.25\left(\mathrm{~s}, 3 \mathrm{H}, \mathrm{CH}_{3}\right) ; 4.00\left(\mathrm{~d}, 1 \mathrm{H}, \mathrm{H}^{1 \mathrm{a}},{ }^{2} \mathrm{JH}^{1 \mathrm{a}}-\mathrm{H}^{1 \mathrm{~b}}=\right.$ 17.20); $5.00\left(\mathrm{~d}, 1 \mathrm{H}, \mathrm{H}^{1 \mathrm{~b}},{ }^{2} \mathrm{JH}^{1 \mathrm{~b}}-\mathrm{H}^{1 \mathrm{a}}=17.20\right) ; 6.05\left(\mathrm{~s}, 1 \mathrm{H}, \mathrm{H}^{4}\right) ; 6.55-7.55(\mathrm{~m}, 17 \mathrm{H}$, Aromatic $\mathrm{H})$; $8.15\left(\mathrm{dd}, 1 \mathrm{H}, \mathrm{H}^{5},{ }^{4} \mathrm{~J}=1.25,{ }^{3} \mathrm{~J}=7.70\right) .{ }^{13} \mathrm{C} \mathrm{NMR}: 21.50\left(\underline{\mathrm{CH}}_{3}\right) ; 55.35\left(\mathrm{~N}_{-} \underline{\mathrm{CH}}_{2}-\right) ; 54.50(-\underline{\mathrm{CH}}-)$; 102.40 (spiro-C); $126.25 ; 128.85 ; 132.75 ; 140.20 ; 140.90 ; 148.70 ; 161.00$ (quat. $\mathrm{C} \mathrm{sp}^{2}$ ); 125.20 ; $125.75 ; 126.25 ; 126.45 ; 127.40 ; 127.50 ; 128.50 ; 128.75 ; 129.25 ; 130.20 ; 134.25 ;$ (tert. C sp ${ }^{2}$ ); $187.65(\mathrm{C}=\mathrm{O})$. MS (EI, $70 \mathrm{eV}):[\mathrm{M}]^{+} \cdot \mathrm{m} / \mathrm{z}: \mathrm{M}(444.5)\left[\mathrm{C}_{30} \mathrm{H}_{24} \mathrm{~N}_{2} \mathrm{O}_{2}\right](15 \%) ; 444(10 \%) ; 207$ (100\%). Anal. Calcd. For $\mathrm{C}_{30} \mathrm{H}_{24} \mathrm{~N}_{2} \mathrm{O}_{2}$ : C, 81.06; H, 5.44; N, 6.30. Found: C, 81.15; H, 5.48; N, 6.17 .

\section{2-Phenyl-4'-phenyl-3'-(4-methoxyphenyl)-1,2-dihydro-4H,4' $H$-spiro[isoquinoline-3,5'-}

isoxazol]-4-one (11). This compound was obtained as white-yellow powder. Yield 57-59\%. M.p. 228-231 ${ }^{\circ} \mathrm{C}$. IR, $v(\mathrm{C}=\mathrm{O}): 1693 \mathrm{~cm}^{-1} .{ }^{1} \mathrm{H}$ NMR: $3.70\left(\mathrm{~s}, 3 \mathrm{H}, \mathrm{CH}_{3}\right) ; 4.00\left(\mathrm{~d}, 1 \mathrm{H}, \mathrm{H}^{1 \mathrm{a}},{ }^{2} \mathrm{JH}^{1 \mathrm{a}}-\mathrm{H}^{1 \mathrm{~b}}=\right.$ 17.60); $4.95\left(\mathrm{~d}, 1 \mathrm{H}, \mathrm{H}^{1 \mathrm{~b}},{ }^{2} \mathrm{JH}^{1 \mathrm{~b}}-\mathrm{H}^{1 \mathrm{a}}=17.60\right) ; 6.00\left(\mathrm{~s}, 1 \mathrm{H}, \mathrm{H}^{4}\right)$; 6.70-7.50 (m, $17 \mathrm{H}$, Aromatic H); $8.20\left(\mathrm{dd}, 1 \mathrm{H}, \mathrm{H}^{5},{ }^{4} \mathrm{~J}=1.30,{ }^{3} \mathrm{~J}=7.75\right) .{ }^{13} \mathrm{C}$ NMR: $55.30(-\underline{\mathrm{CH}}-) ; 55.35\left(\mathrm{NCH}_{2}-\right) ; 102.20$ (spiroC); $1241.50 ; 128.90 ; 132.85 ; 140.90 ; 148.70 ; 160.65 ; 160.95$ (quat. $\mathrm{C} \mathrm{sp}^{2}$ ); 113.95; 125.10; $125.75 ; 126.35 ; 126.50 ; 127.40 ; 127.50 ; 128.45 ; 128.75 ; 129.10 ; 130.25 ; 134.20$ (tert. C sp ${ }^{2}$ ); $187.85(\mathrm{C}=\mathrm{O})$. MS (EI, $70 \mathrm{eV}):[\mathrm{M}]^{+} \cdot \mathrm{m} / \mathrm{z}: \mathrm{M}(460.5)\left[\mathrm{C}_{30} \mathrm{H}_{24} \mathrm{~N}_{2} \mathrm{O}_{3}\right](8 \%) ; 223(100 \%)$. Anal. Calcd. For $\mathrm{C}_{30} \mathrm{H}_{24} \mathrm{~N}_{2} \mathrm{O}_{3}$ : C, 78.24; H, 5.25; N, 6.08. Found: C, 79.05; H, 5.34; N, 6.12.

2-Phenyl-4'-phenyl-3'-(4-chlorophenyl)-1,2-dihydro-4H,4' $H$-spiro[isoquinoline-3,5'-

isoxazol]-4-one (12). This compound was obtained as white-yellow powder. Yield 71\%. M.p. 242-245 ${ }^{\circ}$. IR, $v(\mathrm{C}=\mathrm{O}): 1685 \mathrm{~cm}^{-1} .{ }^{1} \mathrm{H}$ NMR: $4.00\left(\mathrm{~d}, 1 \mathrm{H}, \mathrm{H}^{1 \mathrm{a}},{ }^{2} \mathrm{JH}^{1 \mathrm{a}}-\mathrm{H}^{1 \mathrm{~b}}=17.20\right) ; 4.95(\mathrm{~d}, 1 \mathrm{H}$, $\left.\mathrm{H}^{1 \mathrm{~b}},{ }^{2} \mathrm{JH}^{1 \mathrm{~b}}-\mathrm{H}^{1 \mathrm{a}}=17.20\right) ; 6.05\left(\mathrm{~s}, 1 \mathrm{H}, \mathrm{H}^{4}\right) ; 6.50-7.55\left(\mathrm{~m}, 17 \mathrm{H}\right.$, Aromatic H); $8.20\left(\mathrm{dd}, 1 \mathrm{H}, \mathrm{H}^{5},{ }^{4} \mathrm{~J}\right.$ $\left.=1.35,{ }^{3} \mathrm{~J}=7.60\right) .{ }^{13} \mathrm{C}$ NMR: 54.30 (-CH-); $55.35\left(\mathrm{NCH}_{2}-\right) ; 103.00$ (spiro-C); 132.35; 135.95; 140.90; 148.55; 160.05; (quat. C sp ${ }^{2}$ ); $125.40 ; 125.75 ; 126.55 ; 126.70 ; 127.55 ; 128.55 ; 128.80$; 130.15; 134.35 (tert. $\left.\mathrm{C} \mathrm{sp}^{2}\right) ; 187.35(\mathrm{C}=\mathrm{O})$. MS (EI, $\left.70 \mathrm{eV}\right):[\mathrm{M}]^{+} \cdot \mathrm{m} / \mathrm{z}: \mathrm{M}(464)\left[\mathrm{C}_{29} \mathrm{H}_{21} \mathrm{ClN}_{2} \mathrm{O}_{2}\right]$ (15\%); 466 (8\%); 277 (100\%). Anal. Calcd. For $\mathrm{C}_{29} \mathrm{H}_{21} \mathrm{ClN}_{2} \mathrm{O}_{2}: \mathrm{C}, 74.91 ; \mathrm{H}, 4.55 ; \mathrm{N}, 6.03$. Found: C, 74.65; H, 4.48; N, 6.13.

2-Phenyl-4'-phenyl-3'-(4-nitrophenyl)-1,2-dihydro-4H,4' $\boldsymbol{H}$-spiro[isoquinoline-3,5'-isoxazol] -4-one (13). This compound was obtained as white-yellow powder. Yield 50\%. M.p. 194-197 ${ }^{\circ} \mathrm{C}$. IR, $v(\mathrm{C}=\mathrm{O}): 1696 \mathrm{~cm}^{-1} .{ }^{1} \mathrm{H}$ NMR: $3.85\left(\mathrm{~d}, 1 \mathrm{H}, \mathrm{H}^{1 \mathrm{a}},{ }^{2} \mathrm{JH}^{1 \mathrm{a}}-\mathrm{H}^{1 \mathrm{~b}}=17.25\right) ; 4.60\left(\mathrm{~d}, 1 \mathrm{H}, \mathrm{H}^{1 \mathrm{~b}}\right.$, 
$\left.{ }^{2} \mathrm{JH}^{1 \mathrm{~b}}-\mathrm{H}^{1 \mathrm{a}}=17.25\right) ; 6.00\left(\mathrm{~s}, 1 \mathrm{H}, \mathrm{H}^{4}\right) ; 6.50-7.60(\mathrm{~m}, 17 \mathrm{H}$, Aromatic $\mathrm{H}) ; 8.15\left(\mathrm{dd}, 1 \mathrm{H}, \mathrm{H}^{5},{ }^{4} \mathrm{~J}=\right.$ $\left.1.25,{ }^{3} \mathrm{~J}=7.80\right) .{ }^{13} \mathrm{C}$ NMR: $54.00(-\mathrm{CH}-) ; 55.30\left(\mathrm{NCH}_{2}-\right) ; 102.80$ (spiro-C); 132.55; 136.00; $140.95 ; 148.50 ; 161.00$ (quat. $\mathrm{C} \mathrm{sp}^{2}$ ); $125.40 ; 125.80 ; 126.55 ; 126.85 ; 127.55 ; 128.65 ; 128.85$; 130.20; 134.40 (tert. C sp $\left.)^{2}\right) ; 187.80(\mathrm{C}=\mathrm{O})$. MS (EI, $\left.70 \mathrm{eV}\right)$ : $[\mathrm{M}]^{+} . \mathrm{m} / \mathrm{z}: \mathrm{M}(475)\left[\mathrm{C}_{29} \mathrm{H}_{21} \mathrm{~N}_{3} \mathrm{O}_{4}\right]$ (25\%); 90 (100\%). Anal. Calcd. For $\mathrm{C}_{29} \mathrm{H}_{21} \mathrm{~N}_{3} \mathrm{O}_{4}$ : C, 73.25; H, 4.45; N, 8.84. Found: C, 73.02; H, $4.39 ; \mathrm{N}, 8.57$.

2,3'-Di-phenyl-4' -(4-methylphenyl)-1,2-dihydro-4H,4' $H$-spiro[isoquinoline-3,5'-isoxazol]-4one (14). This compound was obtained as white-yellow powder. Yield $40 \%$. M.p. $260-264{ }^{\circ} \mathrm{C}$. IR, $v(\mathrm{C}=\mathrm{O}): 1695 \mathrm{~cm}^{-1} .{ }^{1} \mathrm{H}$ NMR: $2.20\left(\mathrm{~s}, 3 \mathrm{H}, \mathrm{CH}_{3}\right) ; 4.75\left(\mathrm{~d}, 1 \mathrm{H}, \mathrm{H}^{1 \mathrm{a}},{ }^{2} \mathrm{JH}^{1 \mathrm{a}}-\mathrm{H}^{1 \mathrm{~b}}=17.00\right) ; 4.80$ $\left(\mathrm{d}, 1 \mathrm{H}, \mathrm{H}^{1 \mathrm{~b}},{ }^{2} \mathrm{JH}^{1 \mathrm{~b}}-\mathrm{H}^{1 \mathrm{a}}=17.00\right) ; 6.00\left(\mathrm{~s}, 1 \mathrm{H}, \mathrm{H}^{4}\right) ; 6.90-7.50(\mathrm{~m}, 16 \mathrm{H}$, Aromatic H$) ; 7.60(\mathrm{dd}$, $\left.1 \mathrm{H}, \mathrm{H}^{5},{ }^{4} \mathrm{~J}=1.20,{ }^{3} \mathrm{~J}=7.35\right) .{ }^{13} \mathrm{C}$ NMR: $21.15\left(\underline{\mathrm{CH}}_{3}\right) ; 52.15\left(\mathrm{NCH}_{2}-\right) ; 57.40(-\mathrm{CH}-) ; 103.95$ (spiro-C);108.15; 130.35; 137.30; 139.95; 146.45; 158.45 (quat. $\mathrm{C} \mathrm{sp}{ }^{2}$ ); $125.30 ; 126.40 ; 127.20$; $127.35 ; 127.50 ; 127.75 ; 128.40 ; 129.05 ; 129.30 ; 129.50 ; 129.80 ; 133.55$ (tert. $\left.\mathrm{C} \mathrm{sp}{ }^{2}\right) ; 192.35$ $(\mathrm{C}=\mathrm{O})$. MS (EI, $70 \mathrm{eV}):[\mathrm{M}]^{+} \mathrm{m} / \mathrm{z}: \mathrm{M}(444)\left[\mathrm{C}_{30} \mathrm{H}_{24} \mathrm{~N}_{2} \mathrm{O}_{2}\right](15 \%) ; 207(100 \%)$. Anal. Calcd. For $\mathrm{C}_{30} \mathrm{H}_{24} \mathrm{~N}_{2} \mathrm{O}_{2}$ : C, 81.06; H, 5.44; N, 6.30. Found: $\mathrm{C}, 80.93 ; \mathrm{H}, 5.52 ; \mathrm{N}, 6.14$.

2-Phenyl-3',4'-di-phenyl-1,2-dihydro-4H,4' $H$-spiro[isoquinoline-3,5'-isoxazol]-4-one (15). This compound was obtained as white-yellow powder. Yield $70 \%$. M.p. $202-205{ }^{\circ} \mathrm{C}$. IR, $v$ $(\mathrm{C}=\mathrm{O}): 1703 \mathrm{~cm}^{-1} .{ }^{1} \mathrm{H}$ NMR: $2.20\left(\mathrm{~s}, 3 \mathrm{H}, \mathrm{CH}_{3}\right) ; 2.30\left(\mathrm{~s}, 3 \mathrm{H}, \mathrm{CH}_{3}\right) ; 4.10\left(\mathrm{~d}, 1 \mathrm{H}, \mathrm{H}^{1 \mathrm{aa}},{ }^{2} \mathrm{JH}^{1 \mathrm{a}}-\mathrm{H}^{1 \mathrm{~b}}=\right.$ 17.25); $5.05\left(\mathrm{~d}, 1 \mathrm{H}, \mathrm{H}^{1 \mathrm{~b}},{ }^{2} \mathrm{JH}^{1 \mathrm{~b}}-\mathrm{H}^{1 \mathrm{a}}=17.25\right) ; 6.05\left(\mathrm{~s}, 1 \mathrm{H}, \mathrm{H}^{4}\right) ; 6.45-7.55(\mathrm{~m}, 16 \mathrm{H}$, Aromat $\mathrm{H})$; $8.20\left(\mathrm{dd}, 1 \mathrm{H}, \mathrm{H}^{5},{ }^{4} \mathrm{~J}=1.25,{ }^{3} \mathrm{~J}=7.80\right) .{ }^{13} \mathrm{C}$ NMR: 21.10 and $21.50\left(2 \underline{\mathrm{CH}}_{3}\right) ; 54.35(-\underline{\mathrm{C}} \mathrm{H}-) ; 55.35$ $\left(\mathrm{NCH}_{2}-\right) ; 102.40$ (spiro-C); $126.35 ; 128.90 ; 129.65 ; 136.05 ; 140.10 ; 140.95 ; 148.80 ; 161.10$ (quat. C sp${ }^{2}$ ); $125.15 ; 125.70 ; 126.50 ; 127.50 ; 128.10 ; 128.45 ; 128.60 ; 129.20 ; 130.05 ; 134.20$ (tert. C sp $\left.{ }^{2}\right) ; 187.80(\mathrm{C}=\mathrm{O})$. MS (EI, $\left.70 \mathrm{eV}\right):[\mathrm{M}]^{+} \cdot \mathrm{m} / \mathrm{z}: \mathrm{M}(458)\left[\mathrm{C}_{31} \mathrm{H}_{26} \mathrm{~N}_{2} \mathrm{O}_{3}\right](25 \%) ; 90$ (100\%). Anal. Calcd. For $\mathrm{C}_{31} \mathrm{H}_{26} \mathrm{~N}_{2} \mathrm{O}_{3}: \mathrm{C}, 81.22 ; \mathrm{H}, 5.67 ; \mathrm{N}, 6.11$. Found: $\mathrm{C}, 81.06 ; \mathrm{H}, 5.66 ; \mathrm{N}$, 6.00 .

\section{2-Phenyl-4'-(4-methylphenyl)-3'-(4-methoxyphenyl)-1,2-dihydro-4H,4' $H$-spiro[isoquino-}

line-3,5'-isoxazol]-4-one (16). This compound was obtained as white-yellow powder. Yield 66\%. M.p. $192-195^{\circ} \mathrm{C}$. IR, v (C=O): $1704 \mathrm{~cm}^{-1} .{ }^{1} \mathrm{H}$ NMR: 2.15 (s, 3H, $\left.\mathrm{CH}_{3}\right) ; 3.75\left(\mathrm{~s}, 3 \mathrm{H}, \mathrm{CH}_{3}\right)$; $4.10\left(\mathrm{~d}, 1 \mathrm{H}, \mathrm{H}^{1 \mathrm{aa}},{ }^{2} \mathrm{JH}^{1 \mathrm{a}}-\mathrm{H}^{1 \mathrm{~b}}=17.30\right) ; 5.05\left(\mathrm{~d}, 1 \mathrm{H}, \mathrm{H}^{1 \mathrm{~b}},{ }^{2} \mathrm{JH}^{1 \mathrm{~b}}-\mathrm{H}^{1 \mathrm{a}}=17.30\right) ; 6.00\left(\mathrm{~s}, 1 \mathrm{H}, \mathrm{H}^{4}\right) ; 6.50-$ $7.55\left(\mathrm{~m}, 16 \mathrm{H}\right.$, Aromatic H); $8.20\left(\mathrm{dd}, 1 \mathrm{H}, \mathrm{H}^{5},{ }^{4} \mathrm{~J}=1.15,{ }^{3} \mathrm{~J}=7.70\right) .{ }^{13} \mathrm{C}$ NMR: $21.10\left(\underline{C H}_{3}\right)$; 54.50 (- $\left.\mathrm{OCH}_{3}\right) ; 55.30$ (- $\underline{\mathrm{CH}}$-); $55.35\left(\mathrm{~N}_{\mathrm{CH}}{ }_{2}\right)$ ); 102.15 (spiro-C); 121.60; 128.95; 129.75; 136.10; $140.90 ; 148.80 ; 160.75 ; 160.90$ (quat. $\mathrm{C} \mathrm{sp}{ }^{2}$ ); $113.95 ; 125.05 ; 125.70 ; 126.35 ; 127.50 ; 128.15$; 128.40; 128.60; 129.10; 130.05; 134.15 (tert. $\left.\mathrm{C} \mathrm{sp}^{2}\right) ; 187.95$ (C=O). MS (EI, $\left.70 \mathrm{eV}\right):[\mathrm{M}]^{+} . \mathrm{m} / \mathrm{z}$ : M (474) $\left[\mathrm{C}_{31} \mathrm{H}_{26} \mathrm{~N}_{2} \mathrm{O}_{3}\right](18 \%) ; 90(100 \%)$. Anal. Calcd. For $\mathrm{C}_{31} \mathrm{H}_{26} \mathrm{~N}_{2} \mathrm{O}_{3}$ : C, 78.46; H, 5.52; N, 5.90. Found: C, 77.96; H, 5.43; N, 6.01.

\section{2-Phenyl-4'-(4-methylphenyl)-3'-(4-chlorophenyl)-1,2-dihydro-4H,4' $H$-spiro[isoquino-line-}

3,5'-isoxazol]-4-one (17). This compound was obtained as white-yellow powder. Yield $82 \%$. M.p. 223-225 ${ }^{\circ} \mathrm{C}$. IR, $v(\mathrm{C}=\mathrm{O}): 1710 \mathrm{~cm}^{-1} .{ }^{1} \mathrm{H}$ NMR: $2.20\left(\mathrm{~s}, 3 \mathrm{H}, \mathrm{CH}_{3}\right) ; 4.10\left(\mathrm{~d}, 1 \mathrm{H}, \mathrm{H}^{1 \mathrm{la}},{ }^{2} \mathrm{JH}^{1 \mathrm{~b}}-\right.$ $\left.\mathrm{H}^{1 \mathrm{a}}=17.25\right) ; 5.05\left(\mathrm{~d}, 1 \mathrm{H}, \mathrm{H}^{1 \mathrm{~b}},{ }^{2} \mathrm{JH}^{1 \mathrm{~b}}-\mathrm{H}^{1 \mathrm{a}}=17.25\right) ; 6.05\left(\mathrm{~s}, 1 \mathrm{H}, \mathrm{H}^{4}\right) ; 6.40-7.55(\mathrm{~m}, 16 \mathrm{H}$, Aromat $\mathrm{H}) ; 8.20\left(\mathrm{dd}, 1 \mathrm{H}, \mathrm{H}^{5},{ }^{4} \mathrm{~J}=1.45,{ }^{3} \mathrm{~J}=7.75\right) .{ }^{13} \mathrm{C}$ NMR: $21.10\left(\underline{\mathrm{CH}}_{3}\right) ; 54.10(-\underline{\mathrm{C}} \mathrm{H}-) ; 55.35\left(\mathrm{~N}^{\mathrm{C}} \mathrm{H}_{2}\right)$; 
102.95 (spiro-C); $127.75 ; 129.25 ; 135.90 ; 136.35 ; 140.90 ; 148.65 ; 160.15$ (quat. C sp ${ }^{2}$ ); 125.35 ; $125.75 ; 126.60 ; 127.60 ; 128.30 ; 128.50 ; 128.65 ; 128.80 ; 130.00 ; 134.30$ (tert. $\mathrm{C} \mathrm{sp}{ }^{2}$ ); 187.50 $(\mathrm{C}=\mathrm{O})$. MS (EI, $70 \mathrm{eV}):[\mathrm{M}]^{+} \cdot \mathrm{m} / \mathrm{z}: \mathrm{M}(478)\left[\mathrm{C}_{30} \mathrm{H}_{23} \mathrm{ClN}_{2} \mathrm{O}_{2}\right](15 \%) ; 480$ (8\%); 90 (100\%). Anal. Calcd. For $\mathrm{C}_{30} \mathrm{H}_{23} \mathrm{ClN}_{2} \mathrm{O}_{2}$ : C, 75.23; H, 4.84; N, 5.85. Found: C, 74.96; H, 4.76; N, 5.61.

2-Phenyl-4'-(4-methylphenyl)-3'-(4-nitrophenyl)-1,2-dihydro-4H,4' $\boldsymbol{H}$-spiro[isoquinoline3,5'-isoxazol]-4-one (18). This compound was obtained as white-yellow powder. Yield $72 \%$. M.p. $242-244{ }^{\circ} \mathrm{C}$. IR, $v(\mathrm{C}=\mathrm{O}): 1697 \mathrm{~cm}^{-1} .{ }^{1} \mathrm{H}$ NMR: $2.15\left(\mathrm{~s}, 3 \mathrm{H}, \mathrm{CH}_{3}\right) ; 4.00\left(\mathrm{~d}, 1 \mathrm{H}, \mathrm{H}^{1 \mathrm{a}},{ }^{2} \mathrm{JH}^{1 \mathrm{~b}}-\right.$ $\left.\mathrm{H}^{1 \mathrm{aa}}=17.35\right) ; 4.95\left(\mathrm{~d}, 1 \mathrm{H}, \mathrm{H}^{1 \mathrm{~b}},{ }^{2} \mathrm{JH}^{1 \mathrm{~b}}-\mathrm{H}^{\mathrm{la}}=17.35\right) ; 6.10\left(\mathrm{~s}, 1 \mathrm{H}, \mathrm{H}^{4}\right) ; 6.35-7.75(\mathrm{~m}, 16 \mathrm{H}$, Aromat $\mathrm{H}) ; 8.15$ (dd, $\left.1 \mathrm{H}, \mathrm{H}^{5},{ }^{4} \mathrm{~J}=1.45,{ }^{3} \mathrm{~J}=7.80\right) .{ }^{13} \mathrm{C}$ NMR: $21.10\left(\underline{\mathrm{CH}}_{3}\right) ; 53.70(-\underline{\mathrm{CH}}-) ; 55.40\left(\mathrm{NCH}_{2}-\right)$; 103.95 (spiro-C); $135.45 ; 136.65 ; 140.85 ; 148.25 ; 148.85 ; 159.35$ (quat. $\mathrm{C} \mathrm{sp}{ }^{2}$ ); $123.75 ; 125.60$; $125.75 ; 126.75 ; 127.70 ; 128.25 ; 128.45 ; 128.60 ; 128.75 ; 129.90 ; 134.45$ (tert. C sp ${ }^{2}$ ); 187.00 $(\mathrm{C}=\mathrm{O})$. MS (EI, $70 \mathrm{eV}):[\mathrm{M}]^{+} . \mathrm{m} / \mathrm{z}: \mathrm{M}(489)\left[\mathrm{C}_{30} \mathrm{H}_{23} \mathrm{~N}_{3} \mathrm{O}_{4}\right](25 \%) ; 208$ (100\%). Anal. Calcd. For $\mathrm{C}_{30} \mathrm{H}_{23} \mathrm{~N}_{3} \mathrm{O}_{4}: \mathrm{C}, 73.61 ; \mathrm{H}, 4.74 ; \mathrm{N}, 8.58$. Found: $\mathrm{C}, 72.89 ; \mathrm{H}, 4.56 ; \mathrm{N}, 8.63$.

2-Phenyl-4'-(4-methoxylphenyl)-3'-phenyl-1,2-dihydro-4H,4' $H$-spiro[isoquinoline-3,5'isoxazol]-4-one (19). This compound was obtained as white-yellow powder. Yield 70\%. M.p. 170-173 ${ }^{\circ} \mathrm{C}$. IR, $v(\mathrm{C}=\mathrm{O}): 1697 \mathrm{~cm}^{-1} .{ }^{1} \mathrm{H}$ NMR: $3.85\left(\mathrm{~s}, 3 \mathrm{H}, \mathrm{CH}_{3}\right) ; 4.10\left(\mathrm{~d}, 1 \mathrm{H}, \mathrm{H}^{1 \mathrm{a}},{ }^{2} \mathrm{JH}^{1 \mathrm{a}}-\mathrm{H}^{1 \mathrm{~b}}=\right.$ 17.30); $5.05\left(\mathrm{~d}, 1 \mathrm{H}, \mathrm{H}^{1 \mathrm{~b}},{ }^{2} \mathrm{JH}^{1 \mathrm{~b}}-\mathrm{H}^{1 \mathrm{a}}=17.30\right) ; 6.00\left(\mathrm{~s}, 1 \mathrm{H}, \mathrm{H}^{4}\right) ; 6.50-7.65(\mathrm{~m}, 17 \mathrm{H}$, Aromatic $\mathrm{H})$; 8.05 (dd, $\left.1 \mathrm{H}, \mathrm{H}^{5},{ }^{4} \mathrm{~J}=1.40,{ }^{3} \mathrm{~J}=7.25\right) .{ }^{13} \mathrm{C}$ NMR: $53.80\left(\mathrm{O}-\underline{\mathrm{CH}}_{3}\right) ; 55.20(-\underline{\mathrm{C}} \mathrm{H}-) ; 55.35\left(\mathrm{NCH}_{2}-\right)$; 102.65 (spiro-C); $124.25 ; 127.70 ; 135.90 ; 140.85 ; 148.70 ; 158.35 ; 160.15$ (quat. C sp ${ }^{2}$ ); 113.15 ; $125.35 ; 126.50 ; 127.55 ; 128.45 ; 128.80 ; 131.15 ; 134.25$ (tert. $\left.\mathrm{C} \mathrm{sp}^{2}\right) ; 187.55(\mathrm{C}=\mathrm{O})$. MS (EI, 70 eV): $\left.[\mathrm{M}]^{+} \cdot \mathrm{m} / \mathrm{z}: \mathrm{M}(460)\left[\mathrm{C}_{30} \mathrm{H}_{24} \mathrm{~N}_{2} \mathrm{O}_{3}\right](15 \%) ; 223(100 \%)\right]$. Anal. Calcd. For $\mathrm{C}_{30} \mathrm{H}_{24} \mathrm{~N}_{2} \mathrm{O}_{3}: \mathrm{C}$, 78.24; H, 5.25; N, 6.08. Found: C, 79.01; H, 5.34; N, 5.92.

2-Phenyl-4'-(4-methoxylphenyl)-3'-(4-methylphenyl)-1,2-dihydro-4H,4' $H$-spiro[isoquinoline-3,5'-isoxazol]-4-one (20). This compound was obtained as white-yellow powder. Yield $68 \%$. M.p. $184-186{ }^{\circ} \mathrm{C}$. IR, $v(\mathrm{C}=\mathrm{O}): 1690 \mathrm{~cm}^{-1} .{ }^{1} \mathrm{H}$ NMR: 2.30 (s, $\left.3 \mathrm{H}, \mathrm{CH}_{3}\right) ; 3.65(\mathrm{~s}, 3 \mathrm{H}$, $\left.\mathrm{OCH}_{3}\right) ; 4.10\left(\mathrm{~d}, 1 \mathrm{H}, \mathrm{H}^{1 \mathrm{la}},{ }^{2} \mathrm{JH}^{1 \mathrm{a}}-\mathrm{H}^{1 \mathrm{~b}}=17.30\right) ; 5.05\left(\mathrm{~d}, 1 \mathrm{H}, \mathrm{H}^{1 \mathrm{~b}},{ }^{2} \mathrm{JH}^{1 \mathrm{~b}}-\mathrm{H}^{1 \mathrm{a}}=17.30\right) ; 6.05(\mathrm{~s}, 1 \mathrm{H}$, $\left.\mathrm{H}^{4^{\prime}}\right)$; 6.35-7.55 (m, 16H, Aromatic H); $8.20\left(\mathrm{dd}, 1 \mathrm{H}, \mathrm{H}^{5},{ }^{4} \mathrm{~J}=1.40,{ }^{3} \mathrm{~J}=7.70\right) .{ }^{13} \mathrm{C}$ NMR: 54.00

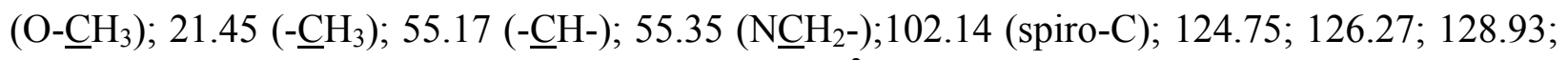
$140.13 ; 140.90 ; 148.83 ; 158.18 ; 161.13$ (quat. $\left.\mathrm{C} \mathrm{sp}^{2}\right) ; 112.99 ; 125.17 ; 125.71 ; 126.39 ; 127.50$; 128.43; 128.70; 129.21; 131.21; 134.17 (tert. $\left.\mathrm{C} \mathrm{sp}^{2}\right) ; 187.85(\mathrm{C}=\mathrm{O})$. MS (EI, $\left.70 \mathrm{eV}\right):[\mathrm{M}]^{+} \mathrm{m} / \mathrm{z}$ : $\mathrm{M}(474)\left[\mathrm{C}_{31} \mathrm{H}_{26} \mathrm{~N}_{2} \mathrm{O}_{3}\right](10 \%) ; 237(100 \%)$. Anal. Calcd. For $\mathrm{C}_{31} \mathrm{H}_{26} \mathrm{~N}_{2} \mathrm{O}_{3}: \mathrm{C}, 78.46 ; \mathrm{H}, 5.52 ; \mathrm{N}$, 5.90. Found: C, 79.03; H, 5.61; N, 5.41.

2-Phenyl-3',4'-(4-methoxylphenyl)-1,2-dihydro-4H,4' $H$-spiro[isoquinoline-3,5'-isoxazol]-4one (21). This compound was obtained as white-yellow powder. Yield $56 \%$. M.p. $198-201^{\circ} \mathrm{C}$. IR, $v(\mathrm{C}=\mathrm{O}): 1698 \mathrm{~cm}^{-1} .{ }^{1} \mathrm{H}$ NMR: $3.65\left(\mathrm{~s}, 3 \mathrm{H}, \mathrm{OCH}_{3}\right) ; 3.75\left(\mathrm{~s}, 3 \mathrm{H}, \mathrm{OCH}_{3}\right) ; 4.10\left(\mathrm{~d}, 1 \mathrm{H}, \mathrm{H}^{\mathrm{la}}\right.$, $\left.{ }^{2} \mathrm{JH}^{1 \mathrm{a}}-\mathrm{H}^{1 \mathrm{~b}}=17.30\right) ; 5.05\left(\mathrm{~d}, 1 \mathrm{H}, \mathrm{H}^{1 \mathrm{~b}},{ }^{2} \mathrm{JH}^{1 \mathrm{~b}}-\mathrm{H}^{1 \mathrm{a}}=17.30\right) ; 6.00\left(\mathrm{~s}, 1 \mathrm{H}, \mathrm{H}^{4}\right) ; 6.35-7.55(\mathrm{~m}, 16 \mathrm{H}$, Aromatic $\mathrm{H}) ; 8.15\left(\mathrm{dd}, 1 \mathrm{H}, \mathrm{H}^{5},{ }^{4} \mathrm{~J}=1.35,{ }^{3} \mathrm{~J}=7.60\right) .{ }^{13} \mathrm{C}$ NMR: 54.15 and $55.20\left(2 \times \mathrm{O}^{-} \mathrm{CH}_{3}\right)$; 55.30 (- $\underline{\mathrm{CH}}-) ; 55.35$ ( $\left.\mathrm{N}_{\mathrm{CH}}{ }_{2}\right)$ ); 101.90 (spiro-C); 121.55; 124.80; 128.95; 140.90; 148.85; 158.20; $160.75 ; 160.90$ (quat. $\mathrm{C} \mathrm{sp}{ }^{2}$ ); $113.00 ; 113.95 ; 125.10 ; 125.70 ; 126.30 ; 127.45 ; 128.40 ; 128.70$; 129.10; 131.20; 134.15 (tert. $\left.\mathrm{C} \mathrm{sp}^{2}\right) ; 188.00(\mathrm{C}=\mathrm{O})$. MS (EI, $\left.70 \mathrm{eV}\right):[\mathrm{M}]^{+} . \mathrm{m} / \mathrm{z}: \mathrm{M}(490)$ 
$\left[\mathrm{C}_{31} \mathrm{H}_{26} \mathrm{~N}_{2} \mathrm{O}_{4}\right](14 \%) ; 253$ (100\%). Anal. Calcd. For $\mathrm{C}_{31} \mathrm{H}_{26} \mathrm{~N}_{2} \mathrm{O}_{4}$ : C, 75.90; H, 5.34; N, 5.71. Found: C, 75.13; H, 5.27; N, 5.65.

\section{2-Phenyl-4'-(4-methoxylphenyl)-3'-(4-chlorophenyl)-1,2-dihydro-4H,4' $H$-spiro[isoquino-}

line-3,5'-isoxazol]-4-one (22). This compound was obtained as white-yellow powder. Yield 78\%. M.p. 200-2003 ${ }^{\circ} \mathrm{C}$. IR, v $(\mathrm{C}=\mathrm{O}): 1695 \mathrm{~cm}^{-1} .{ }^{1} \mathrm{H}$ NMR: 3.65 (s, 3H, $\left.\mathrm{OCH}_{3}\right) ; 4.10(\mathrm{~d}, 1 \mathrm{H}$, $\left.\mathrm{H}^{1 \mathrm{a}},{ }^{2} \mathrm{JH}^{1 \mathrm{a}}-\mathrm{H}^{1 \mathrm{~b}}=17.30\right) ; 5.05\left(\mathrm{~d}, 1 \mathrm{H}, \mathrm{H}^{1 \mathrm{~b}},{ }^{2} \mathrm{JH}^{1 \mathrm{~b}}-\mathrm{H}^{1 \mathrm{a}}=17.30\right) ; 6.05\left(\mathrm{~s}, 1 \mathrm{H}, \mathrm{H}^{4}\right) ; 6.35-7.60(\mathrm{~m}$, $16 \mathrm{H}$, Aromatic H); $8.20\left(\mathrm{dd}, 1 \mathrm{H}, \mathrm{H}^{5},{ }^{4} \mathrm{~J}=1.45,{ }^{3} \mathrm{~J}=7.80\right) .{ }^{13} \mathrm{C} \mathrm{NMR}: 53.90\left(\mathrm{O}_{-} \underline{\mathrm{CH}}_{3}\right) ; 55.20(-$ CH-); 55.35 ( 대 $\left._{2}-\right) ; 102.35$ (spiro-C); 124.60; 128.90; 129.15; 140.90; 148.80;158.20; 161.15 (quat. $\mathrm{C} \mathrm{sp}{ }^{2}$ ); $113.00 ; 125.25 ; 125.70 ; 126.45 ; 127.55 ; 128.50 ; 128.70 ; 129.95 ; 131.20 ; 134.20$ (tert. $\left.\mathrm{C} \mathrm{sp}{ }^{2}\right) ; 187.75(\underline{\mathrm{C}}=\mathrm{O})$. MS (EI, $\left.70 \mathrm{eV}\right):[\mathrm{M}]^{+} \cdot \mathrm{m} / \mathrm{z}: \mathrm{M}(494)\left[\mathrm{C}_{30} \mathrm{H}_{23} \mathrm{ClN}_{2} \mathrm{O}_{3}\right](10 \%) ; 496$ (4\%); 257 (100\%). Anal. Calcd. For $\mathrm{C}_{30} \mathrm{H}_{23} \mathrm{ClN}_{2} \mathrm{O}_{3}$ : C, 72.80; H, 4.68; N, 5.66. Found: C, 71.95; $\mathrm{H}, 4.53 ; \mathrm{N}, 5.58$.

2-Phenyl-4'-(4-methoxylphenyl)-3' -(4-nitrophenyl)-1,2-dihydro-4H,4' $H$-spiro[isoquinoline3,5'-isoxazol]-4-one (23). This compound was obtained as white-yellow powder. Yield 57\%. M.p. $228-231^{\circ} \mathrm{C}$. IR, $v(\mathrm{C}=\mathrm{O}): 1698 \mathrm{~cm}^{-1} .{ }^{1} \mathrm{H}$ NMR: $3.65\left(\mathrm{~s}, 3 \mathrm{H}, \mathrm{OCH}_{3}\right) ; 4.05\left(\mathrm{~d}, 1 \mathrm{H}, \mathrm{H}^{1 \mathrm{a}},{ }^{2} \mathrm{JH}^{1 \mathrm{a}}-\right.$ $\left.\mathrm{H}^{1 \mathrm{~b}}=17.35\right) ; 4.95\left(\mathrm{~d}, 1 \mathrm{H}, \mathrm{H}^{1 \mathrm{~b}},{ }^{2} \mathrm{JH}^{1 \mathrm{~b}}-\mathrm{H}^{1 \mathrm{a}}=17.35\right) ; 6.10\left(\mathrm{~s}, 1 \mathrm{H}, \mathrm{H}^{4^{\prime}}\right) ; 6.40-7.75(\mathrm{~m}, 16 \mathrm{H}$, Aromatic H); 8.15 (dd, $\left.1 \mathrm{H}, \mathrm{H}^{5},{ }^{4} \mathrm{~J}=1.30,{ }^{3} \mathrm{~J}=7.65\right) .{ }^{13} \mathrm{C}$ NMR: $53.40\left(\mathrm{O}-\underline{C H}_{3}\right) ; 55.20(-\underline{C} H-)$; $55.40\left(\mathrm{NCH}_{2-}\right) ; 103.65$ (spiro-C); 123.65; 135.40; 140.85; 148.25; 148.50; 158.45; 159.45 (quat. $\left.\mathrm{C} \mathrm{sp}^{2}\right) ; 113.30 ; 113.90 ; 116.85 ; 123.75 ; 125.65 ; 125.80 ; 126.65 ; 127.70 ; 128.30 ; 128.55 ; 128.85$; 129.25; 131.10; 134.50 (tert. C sp $\left.{ }^{2}\right) ; 187.10(\underline{\mathrm{C}}=\mathrm{O})$. MS (EI, $\left.70 \mathrm{eV}\right):[\mathrm{M}]^{+.} \mathrm{m} / \mathrm{z}: \mathrm{M}(505)$ $\left[\mathrm{C}_{30} \mathrm{H}_{23} \mathrm{~N}_{3} \mathrm{O}_{5}\right](15 \%) ; 208(100 \%)$. Anal. Calcd. For $\mathrm{C}_{30} \mathrm{H}_{23} \mathrm{~N}_{3} \mathrm{O}_{5}: \mathrm{C}, 71.28 ; \mathrm{H}, 4.59 ; \mathrm{N}, 8.31$. Found: C, 70.93; H, 4.48; N, 8.25.

\section{Acknowledgements}

This work was supported by the Programmes "PGR-UMP-BH-2005" and "CUD-BH-2007" Grants from the Ministry of National Education of Morocco. We are indebted to Tuberculosis Antimicrobial Acquisition and Coordinating Facility (TAACF/ USA) for biological tests.

\section{References}

1. Howe, R. K.; Shelton, B. R. J. Org. Chem. 1990, 4603.

2. Smietana, M.; Gouverneur, V.; Mioskowski, C. Tetrahedron Lett. 1999, 40, 1291.

3. Bennani, B.; Kerbal, A.; Ben Larbi, N.; Ben Hadda, T. OMPIC, Moroccan Patent 2004, No. 2769. http://www.taacf.org/publications.htm.

4. Bennani, B.; Kerbal, A.; Ben Larbi, N.; Ben Hadda, T. OMPIC, Moroccan Patent, 2004, No. 2771. http://www.taacf.org/publications.htm. 
5. Benchat, N.; El Bali, B.; Abouricha, S.; Moueqqit, M.; Mimouni, M.; Ben Hadda, T. Chem. Prep. Server, Med./Pharm. Chem. 2003, 1.

6. Anaflous, A.; Benchat, N.; Mimouni, M.; Abouricha, S.; Ben Hadda, T.; El Bali, B.; Hakkou, A.; Hacht, B. Lett. Drug Des. Disc. 2004, 1, 35.

7. Tshiamala, K.; Kerbal, A.; Vebrel, J.; Laude, B. J. Chem. Research (S) 1988, 3, 106.

8. Kerbal, A.; Vebrel, J.; Cerutti, E.; Laude, B. J. Chem. Soc., Chem. Commun. 1989, 632.

9. Filali Baba, B.; Kerbal, A.; Bitit, N.; El Bali, B.; Escudie, J.; Ranaivonjatovo, H.; Bolte, M. Acta. Cryst. 2000, C56, 205.

10. Kerbal, A.; Tshiamala, K.; Vebrel, J.; Laude, B. Bull. Soc. Chim. Belg. 1988, 97, 149.

11. Kerbal, A.; Tshiamala, K.; Verbrel, J.; Laude, B.; Mercier, M. F. Bull. Soc. Chim. Belg. 1991, 2, 159.

12. Theobala, F.; Rodier, N.; Kerbal, A.; Tshiamala, K.; Laude, B. Acta. Cryst. 1990, C46, 1312.

13. Kerbal, A.; Vebrel, J. ; Roche, M. ; Laude, B. Tetrahedron Lett. 1990, 31, 4145.

14. Badri, R.; Kerbal, A.; El Bali, B.; Escudie, B.; Ranaivonjatovo, H.; Bolte, M. Acta. Cryst. 1999, C55.

15. Pivnenko, N. S.; Orlov, V. D.; Nodel'Man, O. A.; Mikhed'Kina, E. I.; Lavrushin, V. F. Zh. Org. Khim. 1980, 16, 1945.

16. Bennani, B.; Filali Baba, B.; EL-Fazazi, A.; Al Houari, G.; El Bali, B.; Bolte, M.; Ben Larbi, N.; Kerbal, A. J. Mar. Chim. Hétéroc. 2003, 1, 1.

17. Akkurt, M.; Yıldırım, S.O.; Badri, R.; Kerbal, A.; Ben Hadda, T.; Büyükgüngör, O. Acta. Cryst. 2006, E62, 5022.

18. Shanmugasundaram, M.; Babu, S. A.; Raghunathan, R.; Malar, E. J. P. Heteroatom Chem. 1999, $10,331$.

19. Raghunathan, R.; Shanmugasundaram, M.; Bhanumathi, S.; Malar, E. J. P. Heteroatom Chem. 1998, 9, 327.

20. Collins, L.; Franzblau, S. G. Antimicrob. Agents Chemother. 1997, 41, 1004.

21. Badri, R.; Kerbal, A.; Najib, B.; El-Bali, B.; Escudie, J.; Ranaivonjatovo, H.; Bolte, M. Acta Cryst. 1999, C55, IUC9900165. 